\title{
Investigations into sampling approaches for chemical analysis of latent fingermark residue
}

\author{
Buddhika N. Dorakumbura ${ }^{a, b \neq}$, Francesco Busetti $^{c}$ and Simon W. Lewis ${ }^{a, b^{*}}$ \\ a School of Molecular and Life Sciences, Curtin University, GPO Box U1987, Perth, Western Australia, 6845, \\ Australia.
}

${ }^{b}$ Curtin Institute of Functional Molecules and Interfaces, Curtin University, GPO Box U1987, Perth, Western Australia, 6845, Australia.

' School of Science, Edith Cowan University, 270 Joondalup Drive, Joondalup, Western Australia, 6027, Australia.

${ }^{\ddagger}$ Present address: ChemCentre, PO Box 1250, Bentley, Western Australia, 6983, Australia.

*Author for correspondence (E-mail: S.Lewis@curtin.edu.au)

\begin{abstract}
The quantitative variation in latent fingermark deposits sampled from the same donor (intra-donor) poses considerable challenges to studies into the chemical composition of latent fingermarks. The work presented here investigates approaches to the sampling of latent fingermark residues within this context. The amount of squalene in fingermarks deposited on non-porous surfaces, determined by GC-MS, was used as an indicator of the amount of non-polar material present. It was found that the percentage difference of squalene between deposits from two hands at a given time, without controlling the deposition force, was in the range of $4-100 \%$. This was reduced to $0-44 \%$ in alternative sampling approaches where deposition force was controlled. These results demonstrate the significant influence of sampling on subsequent chemical analysis of fingermark residues, and offer possible sampling strategies to overcome issues associated with intra-donor variation.
\end{abstract}

Keywords: Fingermark composition, sampling, squalene, GC-MS

\section{Introduction}

The chemical composition of latent fingermarks is subject to change immediately following deposition due to various physical and environmental conditions. While these changes cause many current fingermark detection techniques to become increasingly less effective with time, it has been suggested that they can be useful in ascertaining additional information from the deposit. In this context, significant research into the chemical composition of latent fingermarks has been carried out over the last two decades to (i) infer donor characteristics; ${ }^{1-2}$ (ii) identify potential biomarkers for targeted visualisation; ${ }^{3-4}$ or (iii) estimate the age of the deposit. ${ }^{5-12}$ In particular relation to time-course studies, a key challenge in investigating fingermark compositional variation is the difficulty of obtaining reproducible samples, even from a single donor. ${ }^{6,13}$ Contributing factors include variations in deposition conditions and donor-dependent compositional variation (intra-donor variation). This variability hampers identification of potential ageing trends for specific compound(s), thus hindering the construction of reliable ageing curves proposed to date fingermarks. ${ }^{6,9}$

Recent research has demonstrated that the successful detection of latent fingermarks on non-porous surfaces is less likely than those deposited on porous surfaces. ${ }^{14}$ This is likely due to the minute amount of material deposited and the fragile nature of the deposit. ${ }^{6,9,15-16}$ Relatively little research has been carried 
out to date investigating fingermark degradation over time on non-porous surfaces, presumably due to these restrictive circumstances. ${ }^{3,10-12}$

The first reported study into fingermark degradation process was carried out by Mong et al. in 1999, who reported that comparative quantitation of fingermark compounds over time is not practical because of the compositional variability of subsamples collected from individuals. ${ }^{17}$ Subsequently, Asano et al. investigated the variability of fingermarks collected from three donors in the morning and afternoon on four different days. ${ }^{18}$ They observed little variation in composition due to different sampling times; instead, the major contributing factor was the three different subjects (inter-donor variation). ${ }^{18}$ Similar observations of intradonor variation were made by Archer et al. using deposits from one donor across a day. ${ }^{13}$ Although analysis of variance (ANOVA) did not identify a significant difference, a boxplot of the relative amount of squalene over time indicated there may be an increase in the amount towards the later sampling period.

In a GC-MS investigation of latent fingermark composition, Weyermann et al. cited the lack of reproducibility (both inter-and intra-donor) as the key challenge to applying their approach to the dating of fingermarks. It was found that the percentage relative standard deviations (\% RSDs) for squalene and cholesterol reached up to $80 \%$ for thumbprints collected from a donor within a day over three non-consecutive days. ${ }^{6}$ They subsequently investigated different ways of data processing to identify a parameter that could be plotted against time with minimal intra- and inter-donor variation. Instead of the relative peak area; RPA squalene/internal standard; the use of the ratio of RPA squalene/cholesterol minimised intra-donor variability (from $50 \%$ to $10 \%$ ) of samples obtained from the same day. However, large differences were still detectable between different days $(39 \%){ }^{6}$

Continuing on from this work, Koenig et al. suggested using the ratio of peak area of squalene to the sum of the peak areas of cholesterol and myristyl myristate in order to reduce the \% RSD of intra-variability. Girod and Weyermann later found that donor classification based on "poor" and "rich" lipid donors was affected by the intra-variability to a certain degree. ${ }^{7}$ It was reported that the \% RSD of inter- and intra-variability could be reduced for most of the target lipid compounds by normalising the peak area to the sum of target compounds, excluding squalene. ${ }^{7}$ Squalene showed the highest variability in terms of the relative amount (when normalised to the internal standard) and was thus excluded from the sum of peak areas.

A detailed study into intra-donor variation was carried out by Frick et al. in 2015 where triplicate samples were collected at fixed time intervals from five donors over the course of a day. ${ }^{19}$ For most donors, no clear trend in fingermark composition was observed as a function of time. It was suggested that changes in secretion composition might be unnoticeable as the new secretions would become diluted into secretions currently on the skin surface. Yet chemometric analysis of samples from one donor, who had not handled any food or other substances, gave distinct separation of those collected in the morning and late afternoon. The increase in relative amounts of compounds towards the late afternoon compared to the early sampling periods was attributed to the gradual accumulation of sebum on the skin surface. Investigation of intra-donor variability over one month found variation in the amount of squalene; however, no clear trend was observed across all donors. ${ }^{19}$

In the same study, Frick et al. employed an extensive number of donors ( $n=106)$ to study inter-donor variation, finding that squalene and free fatty acids were the principal compounds responsible for the interdonor variability. ${ }^{19}$ Nevertheless, these differences were not sufficient to enable discrimination of donors based on traits such as age or biological sex. Based on their results, Frick et al. suggested that discrimination of donors based on their fingermark composition is not a viable approach unless intra-donor variation can be minimised. ${ }^{19}$ 
More recently, Gorka et al. investigated the intra- and inter-donor variability of secretion composition using MALDI-MSI as oppose to conventional GC-MS approach. In this preliminary study, a qualitative degree of consistency in terms of secreted molecules was observed in the intra-donor study. Their inter-donor study (using four donors) emphasised the possibility of differentiating individuals based on the fingermark composition. ${ }^{20}$ This study however focused on qualitative analysis of the fingermark residue, with no commentary on quantitative aspects.

The aim of this current work was to identify a fingermark sampling approach that produces the least quantitative variation between two fingermark samples deposited at a given time, using squalene as an indicator of the amount of non-polar material successfully recovered. To this end, four fingermark sampling approaches were investigated with different control measures over the deposition process.

\section{Materials and Methods}

\subsection{Chemicals}

Squalene ( $\geq 98 \%$; Sigma-Aldrich, USA), squalene- $d_{6}$ (98\%; Toronto Research Chemicals, Canada) and dichloromethane ( $\geq 99.9 \%$; Honeywell Inc., USA) were used as received. A stock solution of squalene at a concentration of $10,000 \mathrm{ppm}$ was prepared in dichloromethane (DCM) and diluted to obtain a working solution of $100 \mathrm{ppm}$. A serial dilution was performed, starting with the working solution, to create a set of standard solutions at concentrations of $0.1,0.5,2.5,10.0,25.0$, and $50.0 \mathrm{ppm}$. A standard solution of $20.0 \mathrm{ppm}$ squalene- $\mathrm{d}_{6}$ was also prepared in DCM. All standard solutions were stored below $-18{ }^{\circ} \mathrm{C}$ to avoid degradation and solvent evaporation.

\subsection{Quantification of Squalene}

Quantitation was performed using the ratio of the peak areas of the analyte and of the deuterated standard $\left(20.0 \mathrm{ppm}\right.$ squalene- $\mathrm{d}_{6}$ ). An external calibration curve, plotting ratio against concentration, was obtained by diluting standards in DCM in the concentration range of $0.1-50.0 \mathrm{ppm}$. Concentrations in the samples were calculated by comparing the peak area ratios of the analyte and their corresponding surrogate standard in the fingermark extracts, to the corresponding ratios in the standard solutions. Calibration curves were acquired at the beginning of each batch of samples.

\subsection{Sample Deposition}

Four deposition conditions were tested in this study, which are described in Sampling Protocols 1-4 below. Three donors representing different demographics (Table 1) were employed for this study.

Table 1. Demographics of the fingermark donors.

\begin{tabular}{cccc}
\hline Donor & Age (years) & Biological sex & Cosmetic use \\
\hline 1 & 23 & Female & Regular use of moisturiser and hand cream. \\
2 & 33 & Female & $\begin{array}{r}\text { Regular use of moisturiser, cosmetics, hair oil and hand cream. } \\
\text { Consumes fish oil daily as a nutritional supplement. }\end{array}$ \\
\hline 3 & 53 & Male & $\begin{array}{r}\text { Regular use of an oil blend on hands. Consumes fish oil as a } \\
\text { nutritional supplement every two days. }\end{array}$ \\
\hline
\end{tabular}

For all sampling protocols, after rubbing hands together for 10 seconds, donors placed five fingertips from one hand onto the matte side of an aluminium strip to produce one sample. They were requested to place 
one fingertip at a time, maintaining a constant contact area as much as possible during deposition. Another sample from the other hand was obtained at the same time to produce one sample set. Deposition time was 10 seconds in all cases. Three sample sets (six samples) per donor were obtained within the course of a day, maintaining at least 30 min between each sampling time. Variations between the four sampling protocols are described below.

\subsubsection{Sampling Protocol 1}

Natural fingermarks were obtained on aluminium foil strips. Donors were requested to wash their hands with liquid soap and warm water for about 2 min and allow to air dry. Donors were then asked to engage in their regular activities for at least for $30 \mathrm{~min}$ without washing their hands or handling any food or chemicals before deposition.

\subsubsection{Sampling Protocol 2}

All aluminium strips were slightly folded lengthwise down the centre, then unfolded to make a centreline crease. The strips were placed on a kitchen scale (Soehnle, Germany) covered with aluminium foil to prevent contamination. Donors were asked to place one fingertip at a time on the aluminium strip while preserving the symmetry of contact area along the centreline, maintaining the reading of the scale at $500 \pm 100 \mathrm{~g}$. Pretreatment of the donors' hands prior to deposition was as per Sampling Protocol 1. This process was carried out for both hands, to give a sample set for the left and right hands of each donor. Each sample set was then cut separately along the centreline using a scalpel which was cleaned with DCM before every use. Within each sample set, one piece of the right-hand sample was pooled with another piece of the left-hand sample (Figure 1).

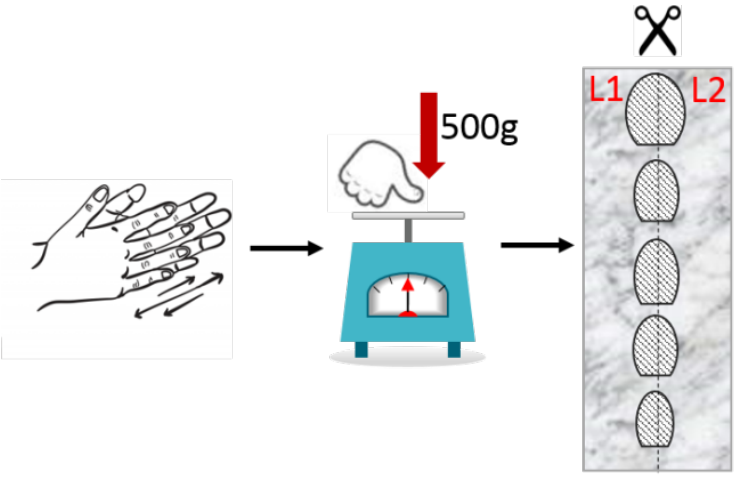

Left hand

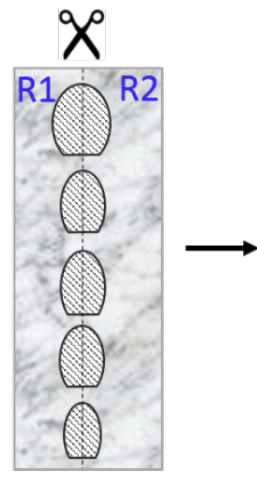

Right hand

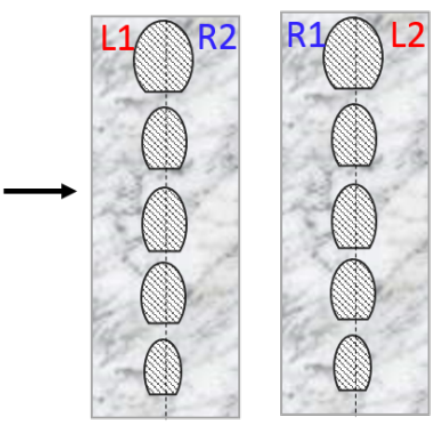

One sample set

Figure 1. Schematic showing the sample deposition procedure for Sampling Protocol 2.

\subsubsection{Sampling Protocol 3}

Sebum-rich deposits were obtained from donors by rubbing their fingers against their nose, forehead, and chin followed by deposition on the substrate. The male participant had no facial hair at any time of sampling. The steps described under section 2.3-Sample Deposition were followed while maintaining the deposition force on the scale as detailed in section 2.3.2.

\subsubsection{Sampling Protocol 4}

Natural fingermarks were obtained from donors as described in Sampling Protocol 1 while maintaining the deposition force on the scale as detailed in section 2.3.2.

\subsection{Sample preparation}

Each aluminium strip (one sample) was rolled and inserted into separate $4 \mathrm{~mL}$ clear glass vials which were pre-rinsed with DCM. Aluminium strips were handled from the edge whilst wearing nitrile gloves to prevent 
sample loss and contamination. A volume of $0.6 \mathrm{~mL}$ of DCM was added followed by $0.1 \mathrm{~mL}$ of squalene- $\mathrm{d}_{6}$ surrogate standard. Extraction vials (Agilent Technologies, USA) were then screw capped using polytetrafluoroethylene-lined caps. The vials were wrapped with aluminium foil to avoid exposure to light during sample extraction and placed in a sonicator bath (Soniclean, Australia) on an orbital shaker (Ratek, Australia) with mild agitation for $10 \mathrm{~min}$. The individual vials were then mixed using vortex mixer (Ratek, Australia) for $2 \mathrm{~min}$. Sample extracts were transferred to $2 \mathrm{~mL}$ amber colour glass crimp top vials (Agilent Technologies, USA) using glass pasteur pipettes (used as received). The GC vials were sealed after placing a piece of aluminium foil (matte side down) over the vial opening. Sample blanks were prepared for every batch of samples using aluminium foil strips bearing no fingermarks. To investigate the extraction efficiency, three $10 \mu \mathrm{L}$ aliquots of $50.0 \mathrm{ppm}$ squalene standard were deposited as individual spots on an aluminium strip and extracted as above. The analysis was carried out in duplicate.

\subsection{Chemical analysis}

Chromatographic analysis was performed using a GC-MS equipped with an autosampler. Instrumental details are described in Table 2.

Table 2. Details of GC-MS instrumentation.

\begin{tabular}{cccc}
\hline Gas chromatograph & Injector & Column & Mass spectrometer \\
\hline Hewlett Packard 6890 & Hewlett Packard & Agilent J\&W DB-1MS UI (60 m x & Hewlett Packard \\
series & 6890 series injector & $0.250 \mathrm{~mm} \mathrm{ID} \mathrm{\times 0.25} \mathrm{\mu m} \mathrm{d}$ ) & $5973 \mathrm{MSD}$ \\
\hline
\end{tabular}

A sample volume of $1 \mu \mathrm{L}$ was injected in splitless mode using an injector maintained at $300^{\circ} \mathrm{C}$ with a purge time of $0.75 \mathrm{~min}$. The $\mathrm{GC}$ oven was programmed from $200^{\circ} \mathrm{C}$, held for $2 \mathrm{~min}$, and then ramped to a final temperature of $300^{\circ} \mathrm{C}$ at a rate of $2.5^{\circ} \mathrm{C} / \mathrm{min}$ and held isothermal for $30 \mathrm{~min}$. The carrier gas was helium with a constant pressure of $24.2 \mathrm{psi}$. The mass spectrometry detector was operated in El mode at $230{ }^{\circ} \mathrm{C}$. The ionisation energy and the electron multiplier voltage were $70 \mathrm{eV}$ and $1576.6 \mathrm{~V}$ respectively. A 9 min solvent delay was applied at the beginning of the chromatographic run, and analytes were measured by scanning in the range $30-550 \mathrm{~m} / \mathrm{z}$ using a single quadrupole mass spectrometer.

\subsection{Data analysis}

The data was processed using MSD ChemStation D.02.00.275 software. Background subtraction was performed for all chromatograms followed by manual integration of the squalene and squalene- $\mathrm{d}_{6}$ peaks. The squalene peak was identified comparing the retention time against the standards and mass spectra against NIST MS library (NIST 98, Agilent Part No. G1033A). The squalene peak area was normalised to that of squalene- $d_{6}$ and the amount of squalene per five fingertips was quantified using the calibration curve. The percentage difference of squalene between left and right hands were then calculated using the following formula:

$\%$ difference of squalene between hands $=\frac{\text { Quantitative difference of } S Q \text { between } \text { hands }}{\text { Average amount of } S Q \text { between both hands }} \times 100$

\section{Results and Discussion}

\subsection{Choice of sample deposition and preparation protocols}

Previous research has reported that studying compositional variation over time using multiple samples obtained at different times is impractical, due to variations in the initial composition of each subsample. ${ }^{6,17}$ This study therefore investigates an alternative approach such that the initial composition of the samples for 
ageing are known within a reasonable margin of certainty. Four fingermark sampling approaches were investigated to identify one resulting in the least quantitative variation between two samples deposited at a given time. The intention was to extract and analyse one sample to characterise the initial composition whilst ageing the other (Figure 2).
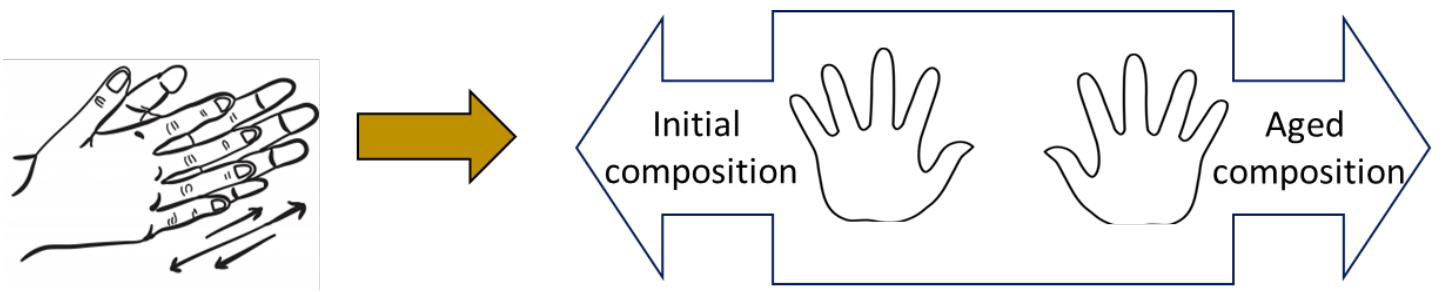

Figure 2. Schematic illustrating the intention of the alternative approach: To obtain two samples at a time with minimum variation and analyse one to know the initial composition. The remainder is to be aged and analysed to characterise the aged composition $(\mathrm{t}=\mathrm{t})$. This will enable knowing the $\mathrm{t}=0$ composition of all samples that are aged.

A fingermark sampling method that provides deposits representative of real-life situations should not require any pre-treatment to fingers or strict controls during sample deposition. However, controlled conditions; such as washing donors' hands, rubbing hands together, charging fingers with sebum, and control of deposition force and time; have been frequently used in fingermark research to preserve sample consistency and increase sensitivity. Most of these studies were related to fingermark visualisation techniques which required deposition of clear, less distorted deposits to assess their performance. Nonetheless, conditions of fingermark sampling employed by various studies vary greatly with little or no explanation as to why certain parameters were chosen.

Two previous studies have reported the use of devices with rigorous control measures; named "fingerprint sampler" ${ }^{21}$ and "Reed-Stanton press rig"22; for research purposes that offered more reproducible fingermarks without distortion caused by changes in the deposition conditions. However, the reproducibility of deposition was assessed by grading the developed fingermarks (by cyanoacrylate fuming ${ }^{21}$ and ink $^{22}$ ), and hence may not be sensitive to ppm level variations in composition. Despite the ongoing debate on the application of strict control measures, such applications were rationalised based on the established research objectives and limitations with analytical conditions.

As non-porous surfaces were of interest for this study, aluminium foil was chosen as a commonly encountered and easy to handle surface. It has been established that only a small amount of fingermark material is transferred onto non-porous surfaces compared to porous surfaces, and that squalene is one of the most abundant lipid compounds found in fingermarks. ${ }^{6,13,15}$ In this study, the use of natural deposits and non-porous substrate as the deposition surface limited the amount of material deposited. Squalene was the only compound that was detectable across all samples by following the given extraction procedure and GCMS analysis. Therefore, the amount of squalene was used as an indicator of the amount of non-polar material successfully extracted by the various sampling protocols.

Sampling Protocol 1 employed natural fingermarks with minimum control over the deposition, which closely resembles deposition events in real-life. Sampling Protocol 2 used rigorous controls over the deposition with the intention of mitigating intra-donor variation due to hand dominance or uneven contact between the fingers and substrates. Charged fingermarks with controlled deposition force were used in Sampling Protocol 3 to investigate what effect this may have on the reproducibility. The use of charged fingermarks is frequently encountered in the literature, predominantly to provide enough material for chemical analysis. ${ }^{3,5-7,9,11,13,15}$ Natural fingermarks obtained as per Sampling Protocol 4 were considered forensically relevant without extreme controls over the deposition. A deposition force of $500 \pm 100 \mathrm{~g}$ exerted by a 
fingertip was chosen as it has been frequently used in literature. ${ }^{5,7-9,22-23}$ Rubbing hands together before deposition has also been frequently performed in previous studies with the aim of distributing material evenly between hands, ${ }^{5,13,24-27}$ although no study to date has investigated the extent of uniformity produced.

Variation caused during sample extraction is another factor that contributes to compositional inconsistencies in final sample extracts. A surrogate standard of squalene- $d_{6}$ was spiked to extraction vials before sonicating the samples to investigate such variations. For an effective sonication extraction, samples must be in contact with the extracting solvent during sonication, ${ }^{28}$ however, the small volume of extracting solvent $(0.7 \mathrm{~mL}$ in total) was not sufficient to coat the rolled aluminium strip completely inside the vial.

Therefore, the sonication bath was placed on an orbital shaker such that the orbital movement of the bath agitated the vials inside, assisting effective extraction. Samples were further mixed in the vortex mixer to achieve extraction of all five deposits. The openings of the GC vials were covered with aluminium foil to prevent extraction of material from the rubber septa. ${ }^{29-31}$

\subsection{Chemical Analysis}

The GC-MS conditions used in this study were initially based on the same reported by Frick et al. ${ }^{19}$ Although their GC-MS program allowed separation of squalene from the fingermark matrix, squalene and squalene- $\mathrm{d}_{6}$ could not be sufficiently resolved. Thus, a long column of $60 \mathrm{~m}$, instead of a $30 \mathrm{~m}$, was used and the GC oven parameters were changed accordingly. Squalene and squalene- $d_{6}$ were then sufficiently resolved (Figure 3).

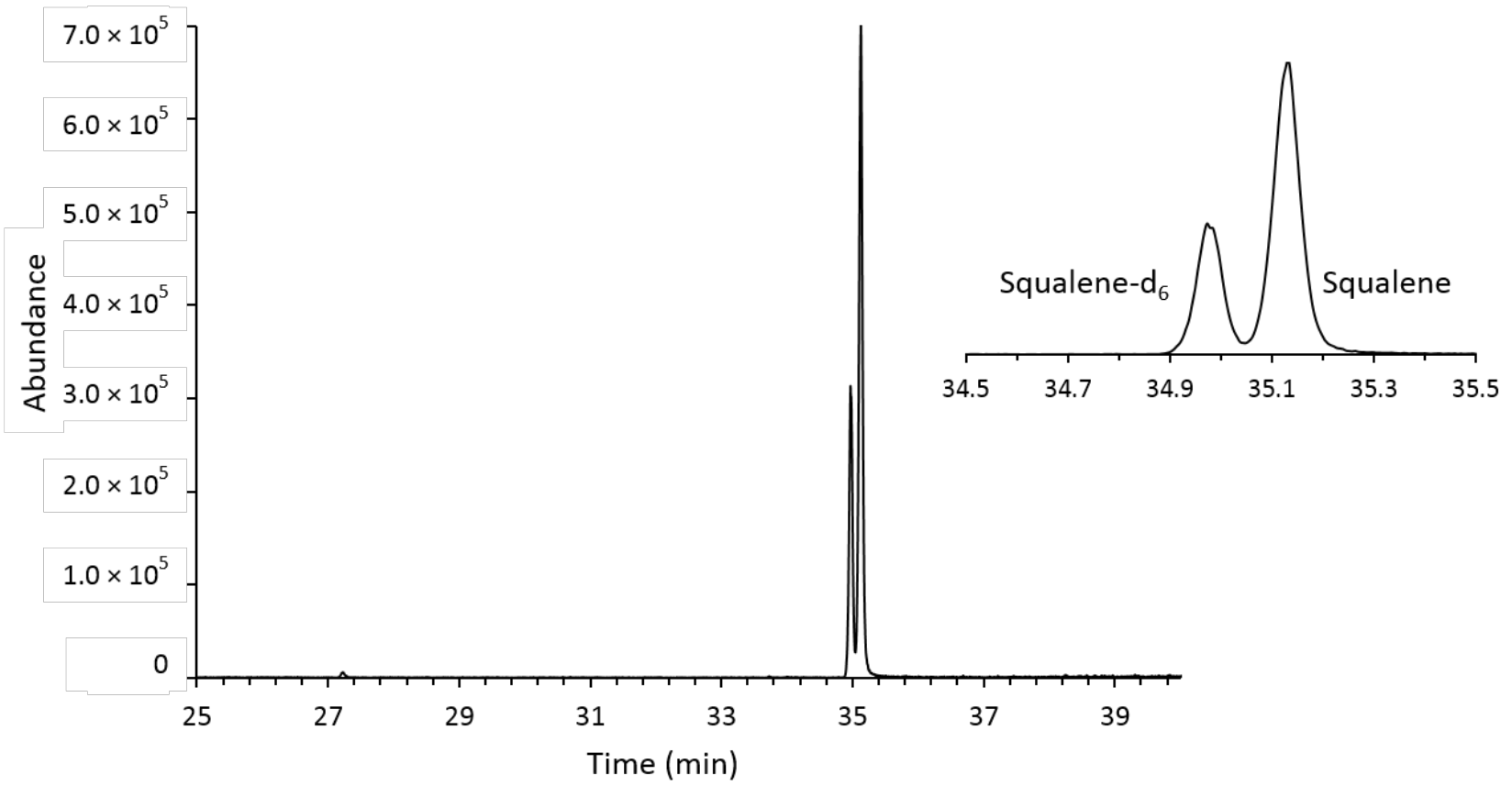

Figure 3. Sample TIC illustrating the separation of squalene and squalene- $d_{6}$ using the modified GC-MS method. 


\subsection{Comparison of proposed sampling protocols}

The average amount of squalene deposited by five fingertips (average between right and left hands) by all three donors in all four sampling protocols are shown in Error! Reference source not found.. In addition, the corresponding percentage differences of the amount of squalene between hands are shown in Figure 5.

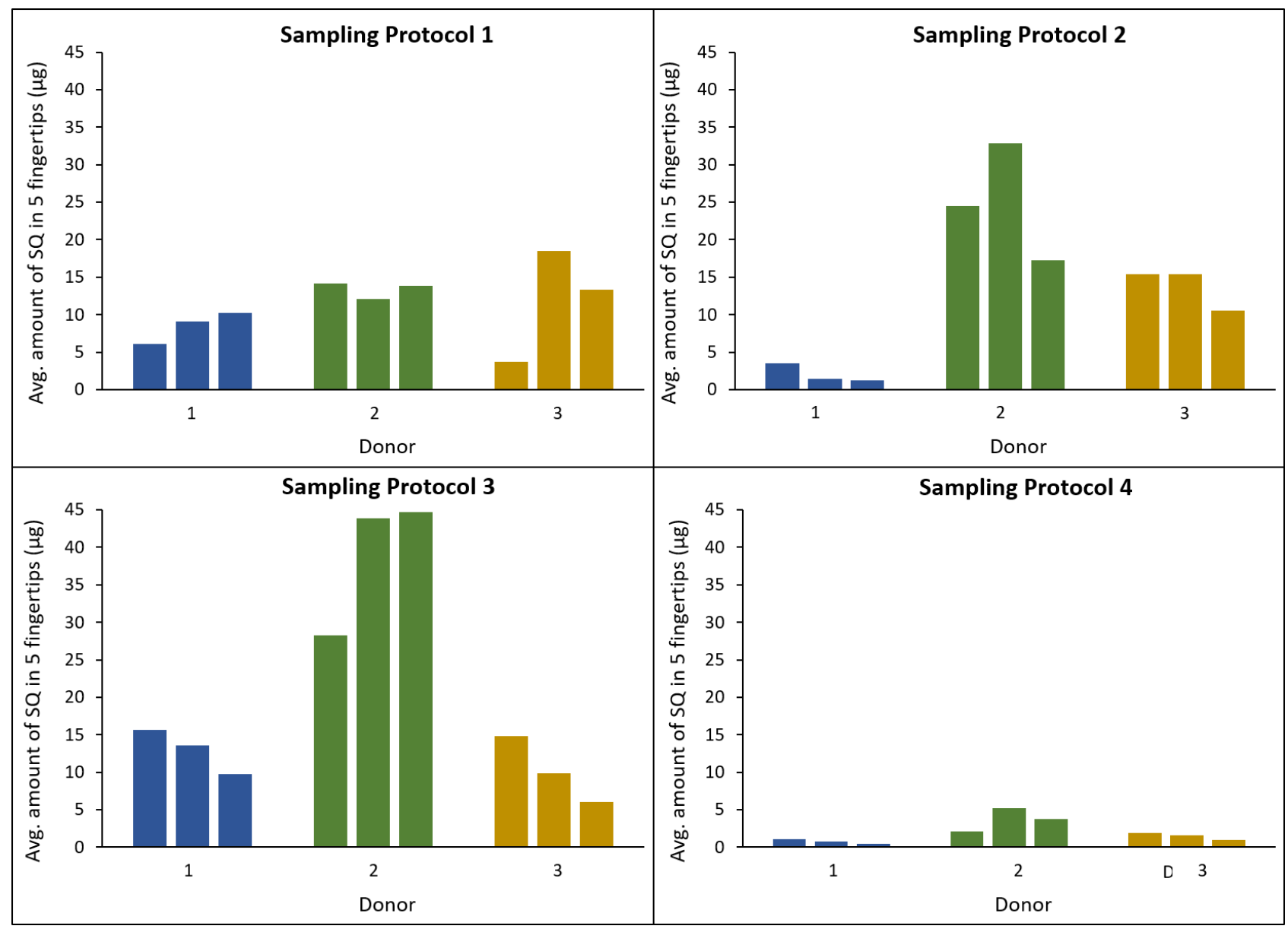

Figure 4. Average amount of squalene between right and left hands (pooled samples in the case of Protocol 2) at a given sampling time in all three donors under the four sampling protocols. From left to right, three coloured bars per each donor represent three sample sets obtained over the course of the day maintaining at least 30 min time interval between consecutive samplings. 


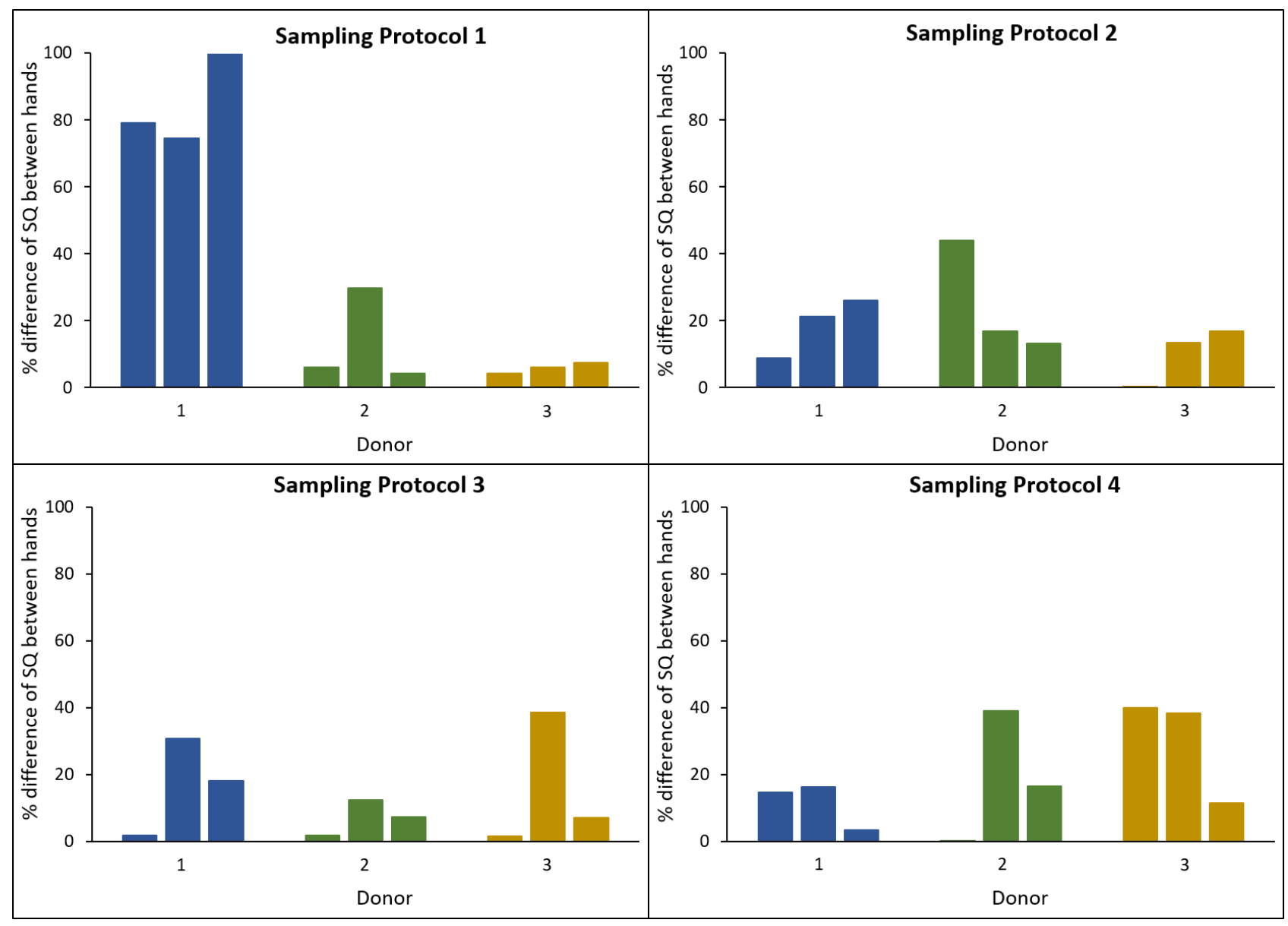

Figure 5. The percentage difference of squalene between hands (between pooled samples for Protocol 2). From left to right, three coloured bars per each donor represent three sample sets obtained over the course of the day maintaining at least 30 min time interval between consecutive samplings.

The average amount of squalene per five fingertips (averaged between both hands) for Sampling Protocols 1-4 was in the range of 3.70-18.44, 1.20-32.86, 6.00-44.67, and 0.49-5.21 $\mu \mathrm{g}$ respectively. Relatively high amounts were detected in Sampling Protocol 3 (where charged deposits were employed) compared to the other sampling protocols, which is consistent with the literature. ${ }^{27}$ Samples from Donor 2 showed the highest amounts, probably due to the regular use of cosmetics in addition to other personal care products. In the study reported by Croxton et al. where natural fingermarks were deposited on Mylar ${ }^{\circledR}$ strips, the amount of squalene per sample (4 fingertips per sample) was $<300 \mathrm{ng},{ }^{27}$ whereas the results reported in this study are in the range of 0.5-44.7 $\mu \mathrm{g}$. While the number of digits deposited per sample in their study was 4 digits instead of 5 digits, the discrepancy in the levels of squalene could be largely due to differences in the extraction procedures and substrates. ${ }^{27}$ In this current study, a recovery percentage of $113 \%$ was achieved for squalene. Although the recovery percentage for derivatised fatty acids was presented in Croxton's previous report, ${ }^{32}$ no such data was available for squalene. Therefore, further comparisons could not be made.

While investigating intra-donor variation over the course of a day at five sampling intervals employing charged deposits from five donors, Frick et al. observed no clear trend in fingermark lipid composition within the 9.00 am-5.00 pm sampling period. ${ }^{19}$ In this present study, a decrease in the amount of squalene in samples obtained between 2.00 pm-4.00 pm from donors 1 and 3 was observed in Sampling Protocol 3. In the same protocol, in samples from donor 2, there was an increase in the first two replicates which were obtained at $10.45 \mathrm{am}$ and $4.30 \mathrm{pm}$ respectively, although the squalene level remained almost the same in the third replicate (at $5.00 \mathrm{pm}$ ). This could be due to the donor activities that contributed to regaining/preserving the squalene concentration during this period. These results indicate that when charged fingermarks are used, the amount of squalene could be influenced by the circadian rhythm of sebaceous 
glands of the forehead, which reaches a maximal amount of sebum production around mid-day. ${ }^{33}$ However, these trends could be masked when total lipid composition is considered because of the further variability of multiple components. Conversely, no clear trend was observed when natural fingermarks were used (Sampling Protocols 1, 2, and 4). This is likely to be caused by random contacts between donors' fingers and surfaces which alter the level of squalene in fingertips, whilst charging fingers immediately prior to deposition minimises such variations. In addition, there was no obvious relationship between the amount of squalene and the dominant hand of the donor, which is in agreement with the observations made by Dominick et al. ${ }^{34}$

High variability in the amount of squalene recovered, between hands, was observed for Sampling Protocol 1, with percentage differences ranging from $4 \%$ to $100 \%$. This is particularly highlighted by the three replicates from Donor 1 as seen in Error! Reference source not found.. There is also a high level of variation between donors, suggesting that both intra-donor and inter-donor variation in the quantity of material deposited is most variable when deposition force is not controlled. The results of the remaining protocols show improved consistency in relation to the percentage difference of squalene recovered for all donors as well as greater reproducibility, with percentage differences ranging from $0 \%$ to $44 \%$. As displayed in Error! Reference source not found., the level of squalene in all replicates of Sampling Protocol 4 was relatively small compared to the other protocols. Thus, manual intergration of peak area over noisy baseline could be a further potential source of variation. The low levels of squalene observed for Sampling Protocol 4 are probably due to natural variation between sampling periods, as Sampling Protocol 2 showed higher levels of squalene despite using broadly the same sampling approach.

Among the four sampling approaches investigated, sample collection as per Sampling Protocol 2 was tedious compared to Sampling Protocol 4, yet displayed only slight improvement in the average percentage difference (2\%). Sampling Protocol 3 yielded the least variation, which suggests charging fingers with sebum prior to deposition can lead to better reproducibility. However, those deposits have a larger amount of material, and may vary in chemical composition, when compared with natural deposits ${ }^{27}$ and thus are not ideal for studying molecular targets in real aged deposits.

The results presented here suggest that Sampling Protocol 4 can reduce variation in the amount of material deposited between two samples and is more suitable for a real-life context. It should be noted that this study was designed to investigate the best approach for sampling of latent fingermarks for chemical analysis and was not aimed at fingermark identification. Using a limited number of donors was necessary to be able to explore more sampling protocols with a reasonable number of replicates per donor. In this study, 72 samples in total were analysed; i. e. 2 samples from both hands at a given time $\times 3$ time intervals $\times 4$ protocols $\times 3$ donors. This experimental design of having a smaller number of donors has been previously reported in fingermark research where the study was not intended to assess the performance of fingermark detection techniques. ${ }^{35}$ The resultant Sampling Protocol 4 is applicable in a larger scale future study which requires two chemically homogeneous fingermark samples (with regards to the lipid content) on non-porous surfaces. Application of this approach to the sampling of fingermarks for time-course experiments and its constructive influence over data interpretation of chromatographic studies will be reported in due course.

\section{Conclusions}

Data interpretation in chromatographic studies of fingermark compositional variation with time has been challenging to date due to differences in the initial composition of subsamples. Despite this, there is a lack of systematic study into the degree of variation of subsamples collected through different sampling methods. The work presented here investigated four different fingermark sampling methods in this context with the intention of suggesting an alternative approach of sampling for time-course experiments. 
The sampling approach taken was found to have a high level of influence on sample reproducibility. Controlling the force during sample deposition reduced the percentage difference of squalene between both hands compared to uncontrolled deposition. Charging fingers with sebum prior to deposition contributed to yield the lowest difference amongst all methods; however, they may not be representative of the aged composition of a latent fingermark. The method outlined under Sampling Protocol 4 is therefore recommended in order to produce two natural deposits with minimal variation in the amount of material deposited at a given time.

\section{Acknowledgments}

The authors would like to acknowledge the contribution of an Australian Government Research Training Program Scholarship in supporting this research. The authors would like to thank all the fingermark donors for their cooperation. Peter Hopper (Curtin University) is acknowledged for his technical expertise with GCMS analysis. Dr Georgina Sauzier (Curtin University) is thanked for reviewing drafts of the manuscript. The authors declare no competing financial interest. This study has been approved by the Curtin University Human Research Ethics Committee (Approval Number RDSE-02-15).

\section{References}

1. Fritz, P., Bronswjik, W., Lepkova, K., Lewis, S., Martin, D., Puskar, L., Infrared microscopy studies of the chemical composition of latent fingermark residues. Microchemical Journal 2013, 111, 40-46.

2. Antoine, K. M.; Mortazavi, S.; Miller, A. D.; Miller, L. M., Chemical differences are observed in children's versus adults' latent fingerprints as a function of time. Journal of Forensic Sciences 2010, 55 (2), 513518.

3. Mountfort, K. A.; Bronstein, H.; Archer, N.; Jickells, S. M., Identification of Oxidation Products of Squalene in Solution and in Latent Fingerprints by ESI-MS and LC/APCI-MS. Analytical Chemistry 2007, 79 (7), 2650-2657.

4. Frick, A.; Chidlow, G.; Goodpaster, J.; Lewis, S.; van Bronswijk, W., Monitoring compositional changes of the lipid fraction of fingermark residues deposited on paper during storage. Forensic Chemistry 2016, 2, 29-36.

5. Koenig, A.; Girod, A.; Weyermann, C., Identification of Wax Esters in Latent Print Residues by Gas Chromatography-Mass Spectrometry and Their Potential Use as Aging Parameters. Journal of Forensic Identification 2011, 61 (6), 606-631.

6. Weyermann, C.; Roux, C.; Champod, C., Initial results on the composition of fingerprints and its evolution as a function of time by GC/MS analysis. Journal of Forensic Sciences 2011, 56 (1), 102-108.

7. Girod, A.; Weyermann, C., Lipid composition of fingermark residue and donor classification using GC/MS. Forensic Science International 2014, 238, 68-82.

8. Girod, A.; Xiao, L.; Reedy, B.; Roux, C.; Weyermann, C., Fingermark initial composition and aging using Fourier transform infrared microscopy ( $\mu$-FTIR). Forensic Science International 2015, 254, 185-196.

9. Girod, A.; Spyratou, A.; Holmes, D.; Weyermann, C., Aging of target lipid parameters in fingermark residue using GC/MS: Effects of influence factors and perspectives for dating purposes. Science \& Justice 2016, 56 (3), 165-180.

10. Muramoto, S.; Sisco, E., Strategies for Potential Age Dating of Fingerprints through the Diffusion of Sebum Molecules on a Nonporous Surface Analyzed Using Time-of-Flight Secondary Ion Mass Spectrometry. Analytical Chemistry 2015, 87 (16), 8035-8038.

11. Pleik, S.; Spengler, B.; Schäfer, T.; Urbach, D.; Luhn, S.; Kirsch, D., Fatty Acid Structure and Degradation Analysis in Fingerprint Residues. Journal of The American Society for Mass Spectrometry 2016, 27 (9), 1565-1574.

12. Wolstenholme, R.; Bradshaw, R.; Clench, M. R.; Francese, S., Study of latent fingermarks by matrixassisted laser desorption/ionisation mass spectrometry imaging of endogenous lipids. Rapid Communications in Mass Spectrometry 2009, 23 (19), 3031-3039.

13. Archer, N. E.; Charles, Y.; Elliott, J. A.; Jickells, S., Changes in the lipid composition of latent fingerprint residue with time after deposition on a surface. Forensic Science International 2005, 154 (2), 224-239. 
14. Chadwick, S.; Moret, S.; Jayashanka, N.; Lennard, C.; Spindler, X.; Roux, C., Investigation of some of the factors influencing fingermark detection. Forensic Science International 2018, 289, 381-389.

15. Frick, A. A. Chemical investigations into the lipid fraction of latent fingermark residue. PhD Thesis, Curtin University, 2015.

16. Girod, A.; Ramotowski, R.; Weyermann, C., Composition of fingermark residue: A qualitative and quantitative review. Forensic Science International 2012, 223 (1), 10-24.

17. Mong, G. M.; Petersen, C.; Clauss, T. Advanced fingerprint analysis project fingerprint constituents; Pacific Northwest National Lab., Richland, WA (US): 1999.

18. Asano, K. G.; Bayne, C. K.; Horsman, K. M.; Buchanan, M. V., Chemical composition of fingerprints for gender determination. Journal of Forensic Science 2002, 47 (4), 1-3.

19. Frick, A.; Chidlow, G.; Lewis, S.; Van Bronswijk, W., Investigations into the initial composition of latent fingermark lipids by gas chromatography-mass spectrometry. Forensic Science International 2015, 254, 133-147.

20. Gorka, M.; Augsburger, M.; Thomas, A.; Bécue, A., Molecular composition of fingermarks: Assessment of the intra- and inter-variability in a small group of donors using MALDI-MSI. Forensic Chemistry 2019, 12, 99-106.

21. Fieldhouse, S., Consistency and reproducibility in fingermark deposition. Forensic Science International 2011, 207 (1), 96-100.

22. Reed, H.; Stanton, A.; Wheat, J.; Kelley, J.; Davis, L.; Rao, W.; Smith, A.; Owen, D.; Francese, S., The Reed-Stanton press rig for the generation of reproducible fingermarks: Towards a standardised methodology for fingermark research. Science \& Justice 2016, 56 (1), 9-17.

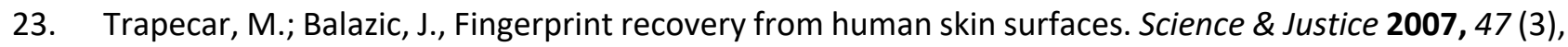
136-140.

24. Fritz, P. Chemical studies into the amino acids present in latent fingermarks. PhD Thesis, Curtin University: Perth, 2015.

25. Cadd, S.; Mota, L.; Werkman, D.; Islam, M.; Zuidberg, M.; De Puit, M., Extraction of fatty compounds from fingerprints for GCMS analysis. Analytical Methods 2015, 7 (3), 1123-1132.

26. Ferguson, L. S.; Wulfert, F.; Wolstenholme, R.; Fonville, J. M.; Clench, M. R.; Carolan, V. A.; Francese, S., Direct detection of peptides and small proteins in fingermarks and determination of sex by MALDI mass spectrometry profiling. Analyst 2012, 137 (20), 4686-4692.

27. Croxton, R. S.; Baron, M. G.; Butler, D.; Kent, T.; Sears, V. G., Variation in amino acid and lipid composition of latent fingerprints. Forensic Science International 2010, 199 (1), 93-102.

28. Snyder, L. R.; Kirkland, J. J.; Glajch, J. L., Practical HPLC method development. John Wiley \& Sons: 2012.

29. Smith, E. D.; Sorrells, K. E., A quantitative comparison of gas chromatograph septum bleed. Journal of Chromatographic Science 1971, 9 (1), 15-17.

30. Olsavicky, V., A comparison of high-temperature septa for gas chromatography. Journal of Chromatographic Science 1978, 16 (5), 197-200.

31. Pattinson, S. J.; Wilkins, J. P., Investigation by gas chromatography-mass spectrometry of potential contamination incurred by the use of crimp-cap vial closures. Analyst 1989, 114 (4), 429-434.

32. Croxton, R. S.; Baron, M. G.; Butler, D.; Kent, T.; Sears, V. G., Development of a GC-MS Method for the Simultaneous Analysis of Latent Fingerprint Components. Journal of Forensic Sciences 2006, 51 (6), 1329-1333.

33. Verschoore, M.; Poncet, M.; Krebs, B.; Ortonne, J.-P., Circadian variations in the number of actively secreting sebaceous follicles and androgen circadian rhythms. Chronobiology International 1993, 10 (5), 349-359.

34. Dominick, A. J.; Welch, L. A.; Daéid, N. N.; Bleay, S. M., Is there a relationship between fingerprint donation and DNA shedding? Journal of Forensic Identification 2009, 59 (2), 133.

35. Acherar, O.; Truong, M. Q.; Robert, S.; Crispino, F.; Moret, S.; Bécue, A., Paper characteristics and their influence on the ability of single metal deposition to detect fingermarks. Forensic Chemistry 2019, 12, 8-24. 